\title{
Mandibular Implant-supported Overdenture as an Occlusion-guide for Maxillary Fixed Implant Prosthesis: A Clinical Report
}

\author{
${ }^{1}$ Pravinkumar G Patil, ${ }^{2}$ Smita Nimbalkar Patil, ${ }^{3}$ Vaibhav Karemore
}

\begin{abstract}
Unlike natural teeth, osseointegrated implants react biomechanically in a different fashion to occlusal load due to lack of the periodontal ligament. Hence, the dental implants may be more prone to occlusal overloading, which is often regarded as one of the potential causes for peri-implant bone loss and failure of the implant/implant prosthesis. Stability of the intercuspal position and reduction of lateral forces are important aspects when providing occlusal equilibration for implantsupported prosthesis. Multiple missing teeth in maxillary and mandibular arches with disturbed occlusal plane due to long-term edentulous condition and overeruption of remaining teeth into the edentulous spaces is the challenging clinical situation. This article describes the management of a patient with multiple missing teeth in both the arches with mandibular implant-supported overdenture and maxillary implant supported fixed prosthesis. The sequence of the treatment planning is highlighted to manage the complex situation to achieve the best predictable occlusion.
\end{abstract}

Keywords: Dental implants, Implant-supported overdentures, Implant restorations, Occlusion, Full mouth rehabilitation.

How to cite this article: Patil PG, Patil SN, Karemore V. Mandibular Implant-supported Overdenture as an Occlusionguide for Maxillary Fixed Implant Prosthesis: A Clinical Report. Int J Oral Implantol Clin Res 2014;5(3):109-113.

Source of support: Nil

Conflict of interest: None

\section{INTRODUCTION}

Planning an occlusion scheme in implant-supported prostheses is a challenging task as the long-term success of the prosthesis principally depends upon it. Unlike natural teeth,

\footnotetext{
${ }^{1}$ Senior Lecturer, ${ }^{2}$ Lecturer, ${ }^{3}$ Assistant Professor

${ }^{1}$ Department of Clinical Dentistry, School of Dentistry, International Medical University, Kuala Lumpur, Malaysia

${ }^{2}$ Department of Orthodontics, Faculty of Dentistry, MAHSA University, Kuala Lumpur, Malaysia

${ }^{3}$ Department of Periodontics, Government Dental College and Hospital, Nagpur, Maharashtra, India

Corresponding Author: Pravinkumar G Patil, Senior Lecturer Department of Clinical Dentistry, School of Dentistry, International Medical University Kuala Lumpur, Malaysia, Phone: 601135022042 e-mail: pravinandsmita@yahoo.co.in
}

osseointegrated implants react biomechanically in a different fashion to occlusal load due to lack of the periodontal ligament. Hence, the dental implants may be more prone to occlusal overloading, which is often regarded as one of the potential causes for peri-implant bone loss and failure of the implant/implant prosthesis. ${ }^{1}$ Once the starting point has been determined and the final outcome is designed, the treatment plan merely becomes the method of reaching the desired result. ${ }^{2}$ Multiple factors needed to be considered in such situations like type of prosthesis (whether removable, fixed or implant supported), occlusal scheme, esthetics, patient desire, patient's financial affordance, etc. The appropriate diagnostic measures like diagnostic mounting, mock preparations, diagnostic wax-ups provide the best possible final prosthetic treatment outcome and help clinicians to manage the situation in simpler way. Stability of the intercuspal position and reduction of lateral force are important aspects for providing occlusal equilibration for implant supported prosthesis. ${ }^{3}$ Anterior guidance and provisional restorations are useful for determination and reconstruction of the occlusal plane. ${ }^{4}$ Managing the single complete denture occlusion is one of the most difficult task as there are numerous clinical challenges in regards to malposed, tipped or supraerupted teeth and uneven occlusal surfaces in the opposing arch. ${ }^{5,6}$ Once the patient's desire inclined toward the implant-supported prostheses, proper sequence of the treatment plan enhances the clinician's comfort. This article describes the management of a patient with multiple missing teeth in both the arches with mandibular implantsupported overdenture and maxillary implant supported fixed prosthesis. The sequence of the treatment planning is highlighted to manage the complex situation to achieve the best predictable occlusion.

\section{CASE REPORT}

A 49-year-old woman was referred to the department of prosthodontics for replacement of missing teeth. Clinical examination revealed partially edentulous maxillary arch with presence of first and second premolars on right and left side; and canine and lateral incisor on right side. Cervical erosive areas were found with maxillary right and left premolars and left canine. Mandibular arch showed canines and 
incisors on both sides showing grade 3 mobility and left third molar with furcation involvement. An orthopantomograph (OPG) examination revealed root pieces with maxillary left second and third molar, severe bone loss with mandibular anterior teeth with lone-standing, extruded mandibular left third molar (Fig. 1). Maxillary teeth were periodontally sound. An extraoral examination revealed senile appearance to the patient due to absence of positive occlusal stop. On the basis of clinical and radiographic examination, the patient was classified as class III (substantially compromised) according to prosthodontic diagnostic index (PDI) resources for partial edentulous patients described by McGarry et al. ${ }^{7}$ Though, the clinical and radiographic findings were suggestive of class II (moderately compromised) PDI, esthetic challenge raised the classification from class II to III. The patient was explained all possible treatment options along with the financial aspects of each. Implant-supported overdenture in mandibular arch and fixed implant prosthesis in maxillary arch was finally planned after discussing all possible treatment options and patient's desire.

\section{Step I: Mandibular Complete Denture to Establish the Occlusal Platform}

Preprosthetic treatment was carried out by extracting all the grade 3 mobile mandibular anterior teeth, a mandibular left third molar and maxillary left second and third molar root pieces. Patient was recalled after 3 months ensuring complete healing of the mandibular edentulous arch. Routine oral prophylaxis of the patient was carried out. Maxillary primary impression was made using an irreversible hydrocolloid impression material (Dentalgin; Prime dental products, Mumbai, India). A diagnostic cast was fabricated from type III gypsum product and mounted on a semi-adjustable articulator (Hanau H2; Teledyne technologies, Los Angeles, California) using the face-bow transfer (Quick Mount FaceBow; Girrbach Corp). Primary and final impressions were made in a conventional manner to obtain the final cast of

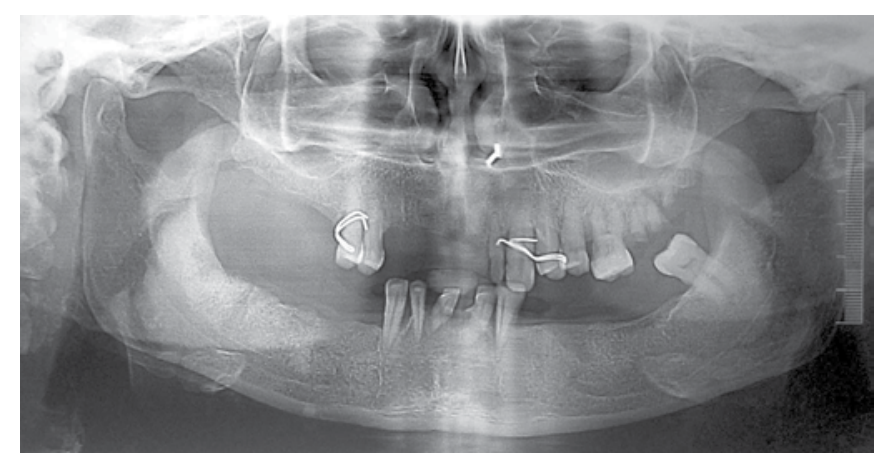

Fig. 1: Pretreatment orthopantomograph. Note the reduced bone support to the mandibular anterior teeth the edentulous mandibular arch. ${ }^{8}$ A temporary record bases were fabricated (on maxillary partial and mandibular complete edentulous cast) with wax-template technique using autopolymerizing polymethylmethacrylate (PMMA). ${ }^{8}$ The record bases were evaluated intraorally and wax occlusion rims were prepared in usual manner. Maxillomandibular relation (MR) was recorded using the wax occlusion rim adjustments. The midline, incisal edge position, occlusal plane, buccal lip support, and anterior segment were indicated on the processed acrylic maxillary base and wax rim. The mandibular edentulous cast was mounted on an articulator with the help of wax-MR. The articulator was programmed using mean horizontal and lateral condylar inclinations (30 and $15^{\circ}$ respectively). The occlusal plane (Curve of Spee and Curve of Wilson) was developed on the mandibular occlusion rim using a Misch occlusal analyzer (Root Laboratory, Leawood, KS, USA) This plate was set against the occlusal surfaces of the mandibular occlusal rim and was based on a 4" sphere. ${ }^{2,9,10}$ Anatomic acrylic resin denture teeth (Acry Rock; Ruthinium, Badia Polesine, Italy) were arranged in class I molar relationship. ${ }^{5}$ The waxed-up maxillary partial and mandibular complete dentures were tried in patient's mouth and evaluated for denture stability, esthetics and occlusion during speech and eccentric jaw movements. Mandibular denture was processed in heat polymerizing acrylic resin (Lucitone 199, Dentsply Intl, New York, PA, USA) with a conventional technique (Fig. 2). ${ }^{11}$ A putty-index (Aquasil Putty; Dentsply, New York, PA, USA) was prepared from maxillary trial denture and mandibular processed denture to use as a guideline to fabricate the maxillary implant restorations.

\section{Step II: Implant Surgical Phase}

Four root form implants (3.75 mm wide and $11.5 \mathrm{~mm}$ long) (Hi-Tech, Herzlia, Israel) were placed in interforaminal region in the mandibular arch following all aseptic norms and surgical principles. The care was taken to place all four

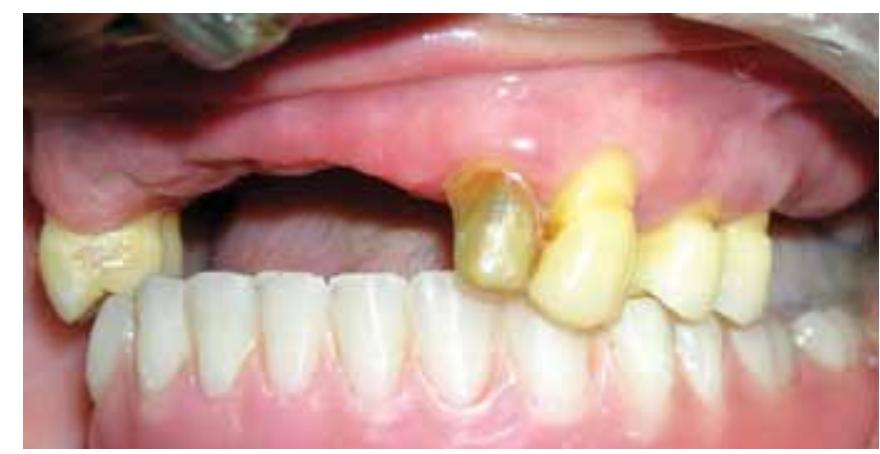

Fig. 2: Mandibular complete denture in place. Note the established denture occlusal table and maxillary remaining teeth making stable centric contacts. Note the level of incisal edges as a guideline for fixed implant restorations in maxillary arch 
implants parallel to each other in all three planes. This was to ensure the all four overdenture ball attachments to lie in one plane to avoid undue stresses during routine placement and removal of the overdenture by the patient. The $4.2 \mathrm{~mm}$ wide and $11.5 \mathrm{~mm}$ long root form implant was placed in maxillary right canine region and $3.75 \mathrm{~mm}$ wide and $11.5 \mathrm{~mm}$ long root form implants were placed in maxillary left and right central incisor. Two $4.2 \mathrm{~mm}$ wide and $8 \mathrm{~mm}$ long implants were placed in maxillary left and right first molar region.

\section{Step III: Implant Prosthetic Phase}

Six months postsurgically, second-stage surgery was performed for all implants in both the arches. After 2 weeks, Dalla-Bona attachments (Hi-Tech; Herzlia, Israel) were attached to four mandibular implants (Fig. 3A). The femalesection of each attachment was carefully attached to the denture by a pick-up-reline method described by Daher (Fig. 3B). ${ }^{12}$ Once the mandibular overdenture was delivered, the procedure for fabricating the multiple unit maxillary fixed prostheses was started. The final impression in maxillary arch was made using impression-transfer-copings, a custom tray, and polyvinylsiloxane. The impression was poured to make a soft tissue cast. The impression of mandibular denture was made to record the occlusal as well as polished surface of the denture to prepare the cast. The MR was recorded in a conventional manner with mandibular denture placed in patient's mouth and remaining maxillary teeth in occlusion. Incisal edges of the mandibular dentureteeth act as a guide for setting the anterior guidance with provision of the most suitable overjet and overbite. The MR was transferred onto the semi-adjustable articulator with the help of facebow and interocclusal records. Maxillary final cast was mounted on the semi-adjustable articulator using face bow and the mandibular cast (prepared from the impression of the polished surface of the denture) was mounted using the MR records. The implant abutments were attached to the fixtures. The implant-abutment preparation was carried out with the arch curvature already established with the mandibular complete denture. The abutments were prepared to achieve parallelism to receive the metalporcelain bridge in anterior region (Figs $4 \mathrm{~A}$ and $\mathrm{B}$ ). The right maxillary incisors and canine and left central incisor fixed
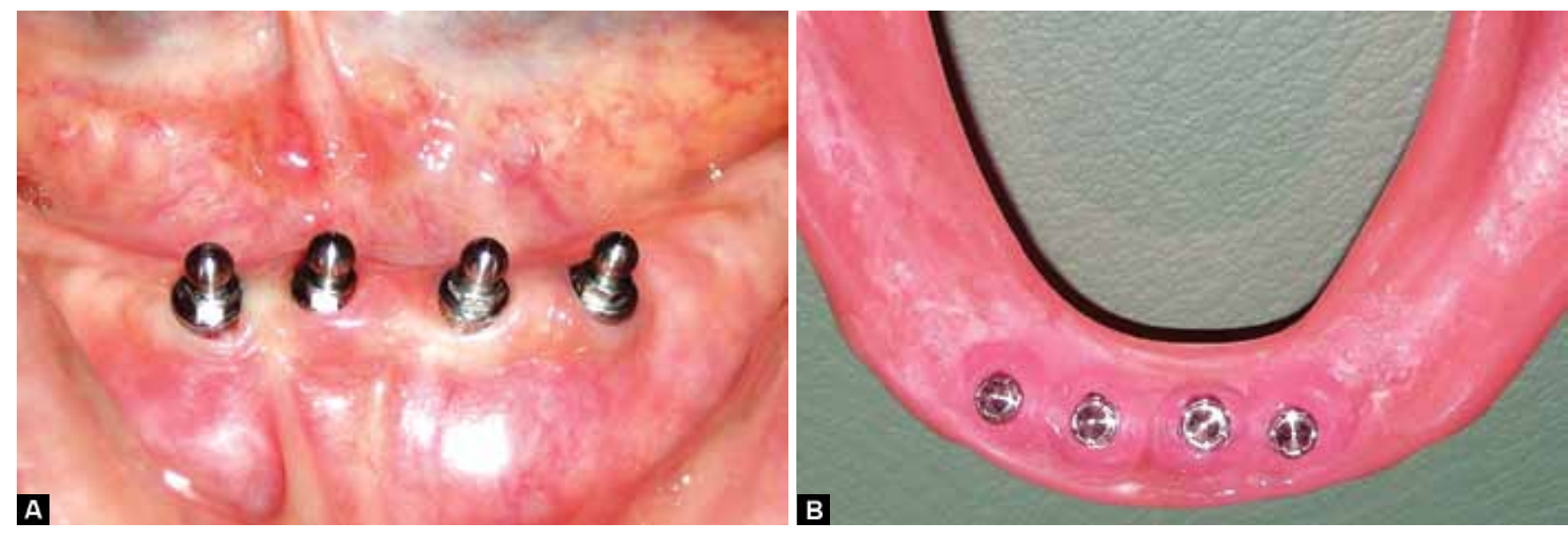

Figs 3A and B: (A) Mandibular arch showing male portion of Dalla-Bona attachments placed parallel to each other and (B) tissue surface of the mandibular denture showing female portion of the Dalla-Bona attachments
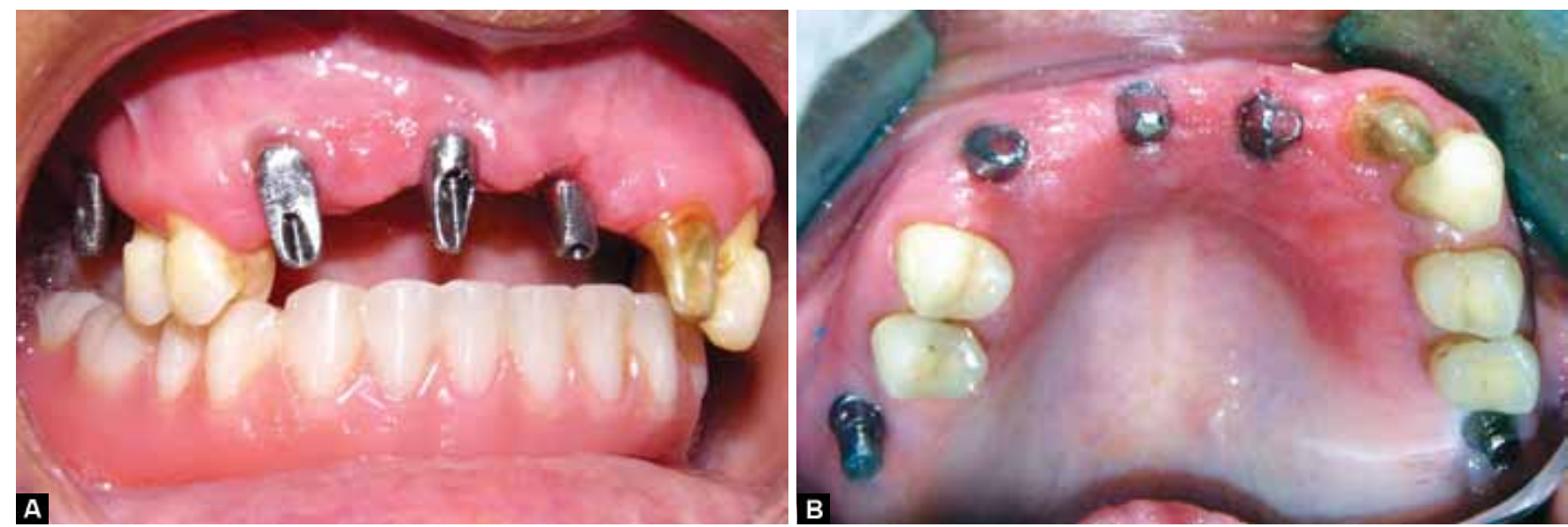

Figs 4A and B: (A) Mandibular denture teeth guiding the implant abutment preparation in maxillary arch and (B) implant abutments in anterior region prepared to achieve the parallelism for a common path 
bridge was fabricated in a conventional way. The implant abutments were then torqued to $30 \mathrm{Ncm}$. The cotton was placed over the abutment screws and sealed with Fermit-N (Vivadent, Schaan, Liechtenstein) in a conventional manner. The maxillary anterior bridge and the molar-crowns were also fabricated and cemented were cemented using NeoTemp (Teledyne Water Pik, Fort Collins, CO, USA). The left lateral incisor was modified to receive the metal ceramic crown as it was already prepared to receive the crown (Fig. 5A). The occlusion, oral hygiene, and soft tissue were re-evaluated 2 weeks after delivery. Post-treatment radiographic evaluation was done by taking the OPG (Fig. 5B). Patient was recalled after 2 months for evaluation of the prostheses. The patient's esthetic improvement was up to the patient's satisfaction. Last recall visit of the patient was 1 year after the treatment, and the patient was pleased with the treatment outcome (Figs 6A and B).

\section{DISCUSSION}

The complex and long standing and long-term edentulism is difficult to manage. Most of the research is related to avoiding failures in implants. This research, in the main, has concentrated on the essential interface between the artificial implant and living bone: osseointegration. The other interface, which is worthy of our full attention, is the one between the implant-supported crown and the antagonist tooth: the occlusion. ${ }^{13}$ The clinical success and longevity of endosteal dental implants are controlled, in a large part, by the mechanical milieu within which they function. The occlusion is a critical component of such a mechanical environment. ${ }^{14}$ Medium or long-term failure of endosseous dental implants after osseointegration, when it has occurred, has been associated in the great majority of cases with occlusal overload. Overload depends ultimately on the number and location of occlusal contacts, which to a great extent are under the clinician's control. ${ }^{15}$ For completely edentulous arches three types of prostheses are available: conventional dentures implant-supported dentures or an implant-retained fixed prosthesis. Though, the satisfaction level is more with the fixed restorations, the implant supported overdenture treatment options can suffice most of the requirements of the fixed restorations as the retention drastically improves in it. Implant-supported overdenture is cost effective option with the added advantage of being
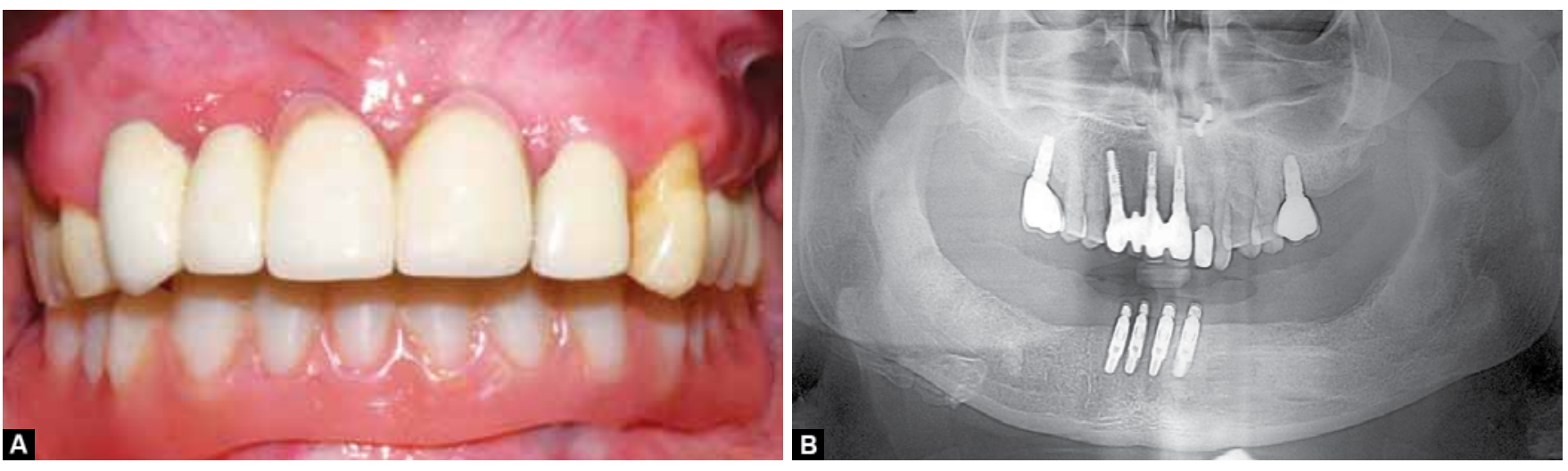

Figs 5A and B: (A) Post-treatment frontal view showing the clinically desirable positioning of the maxillary restorations and (B) post-treatment orthopantomograph
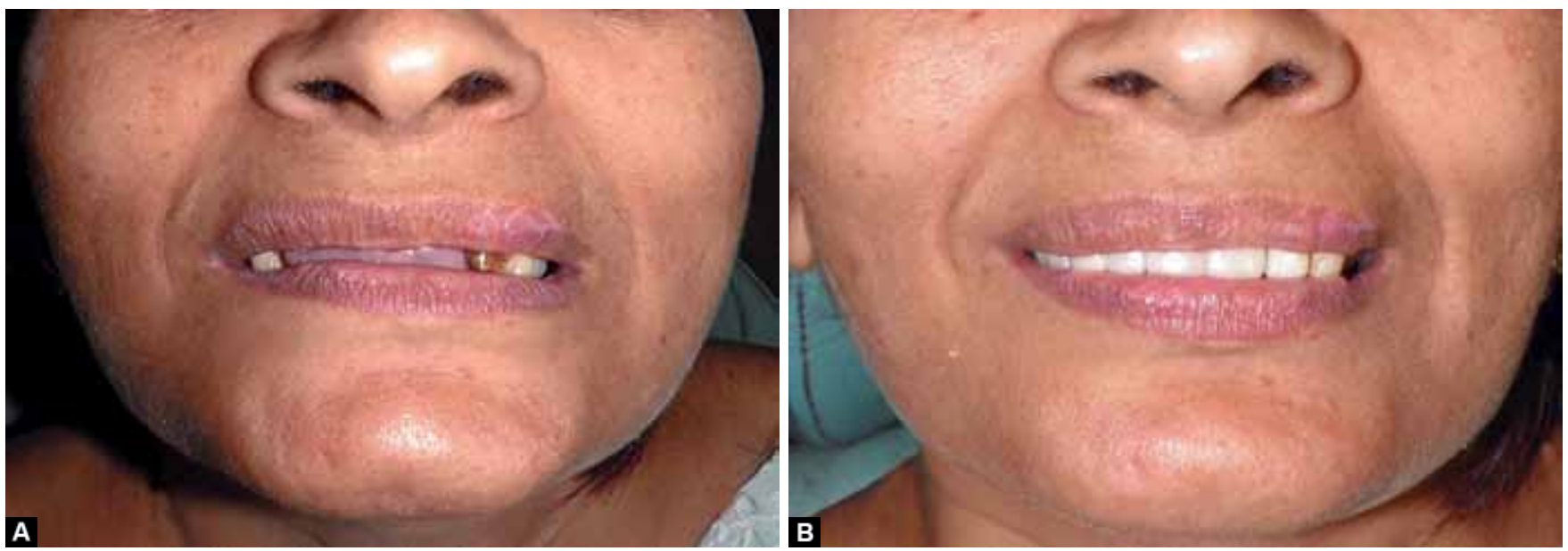

Figs 6A and B: (A) Pretreatment extraoral view showing senile appearance to the patient and (B) post-treatment view. Note the improved esthetic appearance of the patient 
easily cleansable and repairable. The mandibular dentition was restored with a complete denture, because the patient had been wearing, had tolerated, and had accepted this option. Fabricating and placing a mandibular denture with conventional procedures and delivered to the patients to get adapted initially. The attachments are then attached to the denture base with autopolymerizing acrylic resin. ${ }^{12}$ This technique provides a predictable method to achieve a tissuesupported and implant-retained overdenture. ${ }^{12}$ The similar technique has been followed for inserting the overdenture attachments to the existing denture.

Though, the maxillary fixed implant supported prosthesis is set to have occlusion against the occlusal surfaces those are comparatively softer in consistency (acrylic resin) as compared to the ceramic or metal occlusal surfaces. Also the biting force gets reduced with the opposing completed denture than fixed or natural dentition. The occlusal table should be produced in the most suitable zone between tongue and facial tissues to achieve the best possible biomechanical advantage. This zone sometimes needed to be altered for the dental implants as the implant placements are generally directed according to the available bone. To manage the single complete denture, balanced occlusion should be achieved to gain the maximum stability to the denture. The mandibular denture once delivered to the patient the restoring the opposing arch with fixed dental implant prosthesis becomes very easy and predictable. Occlusal rehabilitation with maxillary fixed restoration and mandibular removable denture therapy allows the clinicians to alter the clinical sequence especially during MR procedure. The occlusal relationship can copied with the help of putty indices which can be used to build the porcelain over metal framework and achieve the harmonious occlusion against the overdenture. The diagnostic (preprosthetic) preparations revealed the great help to achieve the best clinical desirable results in such a complicated case. Using a complete denture occlusal platform to achieve the desirable occlusal rehabilitation for the opposing fixed implant prosthesis allows clinician easy way to tackle the complex situations.

\section{REFERENCES}

1. Cabianca M. Combination syndrome: treatment with dental implants. Implant Dent 2003;12(4):300-305.

2. Kim Y, Oh TJ, Misch CE, Wang HL. Occlusal considerations in implant therapy: clinical guidelines with biomechanical rationale. Clin Oral Implants Res 2005;16(1):26-35.

3. Moriwaki Y. A case of occlusal support reconstruction with modification of occlusal plane by fixed prosthetic restoration including dental implant. J Japan Prosthodont Soc 2006 Apr;50(2):288-291.

4. Terakado M. Anterior guidance reconstruction by fixed prosthesis containing bone anchored crown. J Japan Prosthodont Soc 2006 Apr;50(2):292-295.

5. Winkler S. Essentials of complete denture prosthodontics. 2nd ed. St Louis: Ishiyaku Euroamerica; 1994.

6. Patil PG, Parkhedkar RD. Functionally generated amalgam stops for single complete denture: a case report. Dent Res J 2009;6(1):51-54.

7. McGarry TJ, Nimmo A, Skiba JF, et al. Classification system for partial edentulism. J Prosthodont 2002;11(3):181-193.

8. Zarb GA, Finer Y. Identification of shape and location of arch form: the occlusion rim and recording of trial denture base. In: Zarb GA, Bolender CL, Eckert SE, Fenton AH, Jacob RF, Merickske-Stern R, editors. Prosthodontic treatment for edentulous patients: complete dentures and implant supported prostheses. 12th ed. St Louis: Mosby 2005. p. 252-267.

9. Misch CE. Diagnostic casts, preimplant prosthodontics, treatment prostheses, and surgical templates. In: Misch CE, editor. Contemporary Implant Dentistry. 2nd ed. St Louis: Mosby; 1999. p. 137.

10. Dawson PE. The occlusal plane. In: Evaluation, diagnosis, and treatment of occlusal problems. 2nd ed. St Louis: Mosby; 1989. p. 85-91.

11. Morrow RM, Rudd KD, Rhoads JE. Dental laboratory procedures complete dentures. 2nd ed. St Louis: Mosby; 1986. p. 312-338.

12. Daher T. A simple, predictable intraoral technique for retentive mechanism attachment of implant overdenture attachments. J Prosthodont 2003;12(3):202-205.

13. Davies SJ. Occlusal considerations in implantology: good occlusal practice in implantology. Dent Update 2010;37(9): 610-612, 615-616, 619-620.

14. Misch CE, Bidez MW. Implant-protected occlusion: a biomechanical rationale. Compendium 1994;15(11):1330, 1332, 1334 passim; quiz 1344.

15. Rilo B, da Silva JL, Mora MJ, Santana U. Guidelines for occlusion strategy in implant-borne prostheses: a review. Int Dent J 2008;58(3):139-145. 